\title{
A Generic Framework for Local Search: Application to the Sudoku Problem
}

\author{
T. Lambert ${ }^{1,2}$, E. Monfroy ${ }^{1,3, \star}$, and F. Saubion ${ }^{2}$ \\ ${ }^{1}$ LINA, Université de Nantes, France \\ Firstname.Name@lina.univ-nantes.fr \\ ${ }^{2}$ LERIA, Université d'Angers, France \\ Firstname.Name@univ-angers.fr \\ ${ }^{3}$ Universidad Santa María, Valparaíso, Chile \\ Firstname.Name@inf.utfsm.cl
}

\begin{abstract}
Constraint Satisfaction Problems (CSP) provide a general framework for modeling many practical applications. CSPs can be solved with complete methods or incomplete methods. Although some frameworks has been designed to formalized constraint propagation, there are only few studies of theoretical frameworks for local search. In this paper, we are concerned with the design of a generic framework to model local search as the computation of a fixed point of functions and to solve the Sudoku problem. This work allows one to simulate standard strategies used for local search, and to design easily new strategies in a uniform framework.
\end{abstract}

\section{Introduction}

Sudoku literally means single number in Japanese and has reached recently an international popularity. The success of this great puzzle game probably comes from the simplicity of its rules : place digits between $1-9$ on a $9 \times 9$ grid such that each digit appears once in each row, column and each $3 \times 3$ sub-grid. This problem can obviously be considered as a Constraint Satisfaction Problem (CSP), usually defined by a set of variables associated to domains of possible values and by a set of constraints.

Local search techniques [1] have been successfully applied to various combinatorial optimization problems (scheduling, timetabling, transportation ...). In the CSP solving context, local search algorithms are used either as the main resolution technique or in cooperation with other resolution processes (e.g., constraint propagation) [3, 6]. Unfortunately, the definitions and the behaviors of these algorithms are often strongly related to specific implementations and problems.

Usual constraint propagation techniques have already been used to solve Sudoku and in this paper, we are interested in using various local search techniques to solve this problem. Since we want to test different resolution heuristics, our purpose is to use a generic framework based on basic functions in order to provide uniform framework which help to better understand existing local search algorithms and to design new ones.

\footnotetext{
* The author has been partially supported by the Chilean National Science Fund through the project FONDECYT N ${ }^{\circ} 1060373$.
} 
In [7] we have extended the mathematical framework of K.R. Apt [2] to take into account hybridizations of constraint propagation with local search algorithms. The purpose of this paper is to focus on the modeling of basic local search processes and then to improve this previous work by providing a more comprehensive definition to local search algorithms. To obtain a finer definition of local search, we propose a specific computation structure and define the basic functions that will be used iteratively on this structure to create a local search process. Processes are abstracted at the same level by some homogeneous functions called reduction functions. The result of local search is then computed as a fixed point of this set of functions.

The paper is organized as follows : in Section 2 we recall basic definitions related to CSP and local search algorithms. In Section 3 we describe our framework by defining its main components. In Section 4 we provide the operational semantics as the computation of a fixed point of functions over the ordered structure, which corresponds to the instantiation of the generic iteration algorithm proposed by K.R. Apt. Popular local search algorithms and strategies are then designed and applied to solve Sudoku in Section 5 before concluding in Section 6 .

\section{Solving CSP with Local Search}

A CSP is a tuple $(X, D, C)$ where $X=\left\{x_{1}, \cdots, x_{n}\right\}$ is a set of variables taking their values in their respective domains $D=\left\{D_{1}, \cdots, D_{n}\right\}$. A constraint $c \in C$ is a relation $c \subseteq D_{1} \times \cdots \times D_{n}$. In order to simplify notations, $D$ will also denote the Cartesian product of $D_{i}$ and $C$ the union of its constraints. A tuple $d \in D$ is a solution of a $\operatorname{CSP}(X, D, C)$ if and only if $\forall c \in C, d \in c$. In this paper, we always consider finite domains.

Given an optimization problem (which can be minimizing the number of violated constraints and thus trying to find a solution to the CSP), local search techniques [1] aim at exploring the search space, moving from a sample to one of its neighbors. These moves are guided by a fitness function that evaluates the benefit of such a move in order to reach a local optimum.

For the resolution of a $\operatorname{CSP}(X, D, C)$, the search space can be often defined as the set of possible tuples of $D=D_{1} \times \cdots \times D_{n}$ and the neighborhood is a mapping $\mathcal{N}: D \rightarrow 2^{D}$. This neighborhood function defines indeed the possible moves from a sample of $D$ to one of its neighbors and therefore fully defines the exploration landscape. The fitness (or evaluation) function eval is related to the notion of solution and can be defined as the number of constraints $c$ that are not satisfied by the current sample. The problem to solve is then a minimization problem. Given a sample $d \in D$, two basic cases can be identified in order to continue the exploration of $D$ : intensification (choose $d^{\prime} \in \mathcal{N}(d)$ such that eval $\left.\left(d^{\prime}\right)<\operatorname{eval}(d)\right)$ and diversification(choose any other neighbor $d^{\prime}$ ). Any local search algorithm is based on the management of these basic heuristics by introducing specific control features. Here, we abstract moves and neighborhood by functions computing over a given ordered structure. 


\section{A Computational Framework}

In this section, we define the computation structure we will use to represent local search states, together with the functions that will be required to model local search as a fixed point computation.

\subsection{The Computation Structure}

As we have seen, local search acts usually on a structure which corresponds to points of the search space. Here, we propose a more general and abstract definition based on the notion of sample, already suggested.

Definition 1 (Sample). Given a $C S P(X, D, C)$, a sample function is a function $\varepsilon: D \rightarrow 2^{D}$. By extension, $\varepsilon(D)$ denotes the set $\{\varepsilon(d) \mid d \in D\}$.

Generally, $\varepsilon(d)$ is restricted to $d$ and $\varepsilon(D)=D$, but it can also be a scatter of tuples around $d$, or a box of tuples covering $d$. Indeed, the search space $D$ is abstracted by $\varepsilon(D)$ to be used by the local search. Note that in any case, $\varepsilon(D)$ is finite since we consider $D$ to be finite.

The general process of local search can be abstracted by two stages: generate the neighborhood of the current sample and move from the current sample to one of the previously computed neighbor. We define then a local search path as a tuple of samples which represents the path already constructed by the local search process.

Definition 2 (Local Search Path). A local search path $p$ is a finite sequence $\left(s_{1}, \cdots, s_{n}\right)$ such that $\forall 1 \leq i \leq n, s_{i} \in \varepsilon(D)$.

We denote by $P_{\varepsilon(D)}$ the set of all possible local search paths on $\varepsilon(D)$. Given a tuple $p=\left(s_{1}, \cdots, s_{n}\right) \in \varepsilon(D)^{n}$, and an element $s \in \varepsilon(D)$, we denote $p^{\prime}=p \oplus s$ the tuple $\left(s_{1}, \cdots, s_{n}, s\right)$. To simplify notation, we denote $s \in p$ the fact that a sample $s$ is a component of a path $p$.

We now define orderings on this structure. From a practical point of view, a local search process aims at building a finite path whose length is either determined by the fact that a solution has been reached or that a maximum number of iterations have been performed. Therefore, our orderings take into account these two main aspects of local search.

Definition 3 (Ordering on paths). Given $p=\left(s_{1}, \cdots, s_{n}\right)$ and $p^{\prime}=\left(s_{1}^{\prime}, \cdots, s_{m}^{\prime}\right)$ two paths of $P_{\varepsilon(D)}, p \sqsubseteq p^{\prime}$ iff $s_{m}^{\prime} \in \operatorname{Sol}_{\varepsilon(D)}$ or $s_{n} \notin \operatorname{Sol}_{\varepsilon(D)}$ and $m \geq n$.

In order to handle simultaneously paths and neighborhoods, we first define the notion of local search configuration.

Definition 4 (Local Search Configuration). A local search configuration $\mathcal{C}_{\varepsilon(\mathcal{D})}$ is a pair $(p, V)$ where $p=\left(s_{1}, \cdots, s_{n}\right)$ is a local search path and $V \subseteq 2^{\varepsilon(D)}$.

$\mathcal{C}$ is the set of all configurations. The ordering on path is obviously extended to configurations taking into account set inclusion for the neighborhood. 


\subsection{Reduction Function Definitions}

Our definition of reduction functions is based on K.R. Apt's framework [2].

Definition 5 (Reduction function on a structure). Given a partial ordering $(D, \sqsubseteq)$, a reduction function $f$ is a function from $D$ to $D$ which satisfies the following properties:

$-\forall x \in D, x \sqsubseteq f(x)$ (inflationary)

$-\forall x, y \in D, x \sqsubseteq y \Rightarrow f(x) \sqsubseteq f(y)$ (monotonic)

As previously described, the basic components to build a single local search path are move and neighborhood computation.

Definition 6 (Move Function). A move function is a function $\mu: \mathcal{C} \rightarrow \mathcal{C}$ such that $\mu(p, V)=\left(p^{\prime}, \emptyset\right)$ where $p^{\prime}=p \oplus s$ with $s \in V$ if $p=p^{\prime \prime} \oplus s^{\prime}$ and $s^{\prime} \notin \operatorname{Sol}_{\varepsilon(D)}$ and $V \neq \emptyset, p^{\prime}=p$ otherwise.

Definition 7 (Neighborhood Function). A neighborhood function is a function $\nu: \mathcal{C} \rightarrow \mathcal{C}$ with $\nu(p, V)=\left(p, V \cup V^{\prime}\right)$ such that $V^{\prime} \subseteq \varepsilon(D)$ and $V^{\prime} \cap V=\emptyset$.

Move and neighborhood functions are reduction functions.

\subsection{Restricting Functions to Match a Practical Framework}

As mentioned before, we must remark that stop conditions are always added in local search algorithms in order to insure termination. These conditions are basically based on a maximum number of allowed search steps or on a notion of solution (if this notion is available). In the context of CSP solving, this notion of solution has been clearly defined and is taken into account in the definition of our computation structure. Here the maximum number of operations will be defined by a maximum number $\sigma$ of steps in each path (maximal length of a path). Given a move or neighborhood function $f: \mathcal{C} \rightarrow \mathcal{C}$, we define its restriction $f^{\sigma}: \mathcal{C} \rightarrow \mathcal{C}$ as: $f^{\sigma}(p, V)=f(p, V)$ if $|p| \leq \sigma$ and $f^{\sigma}(p, V)=(p, V)$ otherwise.

We must insist on the fact that, after these practical restrictions, we only consider $P$ as the set of all possible local search paths of size $\sigma$. Therefore, this set is finite and $\mathcal{C}$ is also finite. Note that only the restriction concerning $\sigma$ is required to insure finiteness of the structures which is needed to fit the generic iteration framework (section 4).

\section{Local Search as a Fixed Point of Reduction Functions}

In our framework, local search will be described as a fixed point computation on the previously ordered structure. 


\subsection{Chaotic Iterations}

In 2, K.R. Apt proposed the chaotic iteration framework, a general theoretical framework for computing limits of iterations of a finite set of functions over a partially ordered set. In this paper, we do not recall all the theoretical results of K.R. Apt, but we just give the GI algorithm for computing fixed point of functions. Consider a finite set $F$ of functions, and $d$ an element of a partially ordered set $\mathcal{D}$.

\section{GI: Generic Iteration Algorithm}

$d:=\perp$;

$G:=F$;

While $G \neq \emptyset$ do

$$
\begin{aligned}
& \text { choose } g \in G ; \\
& G:=G-\{g\} \\
& G:=G \cup \text { update }(G, g, d) ; \\
& d:=g(d) ;
\end{aligned}
$$

Endwhile

where $\perp$ is the least element of the partial ordering $(\mathcal{D}, \sqsubseteq), G$ is the current set of functions still to be applied $(G \subseteq F)$, and for all $G, g, d$ the set of functions update $(G, g, d)$ from $F$ is such that:

P1 $\{f \in F-G \mid f(d)=d \wedge f(g(d)) \neq g(d)\} \subseteq$ update $(G, g, d)$.

P2 $g(d)=d$ implies that update $(G, g, d)=\emptyset$.

P3 $g(g(d)) \neq g(d)$ implies that $g \in \operatorname{update}(G, g, d)$

Suppose that all functions in $F$ are reduction functions as defined before and that $(\mathcal{D}, \sqsubseteq)$ is finite (note that finiteness is important as is has already been mentioned for our structure) then every execution of the GI algorithm terminates and computes in $d$ the least common fixed point of the functions from $F$ (see [2]).

We now use the GI algorithm to compute the fixed point of our functions. The algorithm is thus feed with:

- a set of move and neighborhood functions, that compose the set $F$,

$-\perp=\emptyset \in P_{\varepsilon(D)}$ to instantiate initial $d$,

- the ordering that we use is the ordering $\sqsubseteq$ on $P_{\varepsilon(D)}$.

In our context, the algorithm terminates and computes the least common fixed point of the functions from $F$, i.e., the result of the whole local search. Inspired by [2, the proof partially relies on an invariant $\forall f \in F-G, f(d)=d$ of the "while" loop in the algorithm. This invariant is preserved by our characterization of the update function. Moreover, since we keep a finite partial ordering and a set of monotonic and inflationary functions, the results of K.R. Apt can be extended here. 


\section{Solving the Sudoku}

As mentioned in the introduction, the Sudoku problem consists in filling a $9 \times 9$ grid so that every row, every column, and every $3 \times 3$ box contains the digits from 1 to 9. Although Sudoku, when generalized to $n^{2} \times n^{2}$ grids to be filled in by numbers from 1 to $n^{2}$ is NP-complete, the popular $9 \times 9$ grid with $3 \times 3$ regions is not difficult to solve with a simple computer program. Therefore, in order to increase the difficulty, we consider $16 \times 16$ grids (published under the name "super Sudoku"), $25 \times 25$ and $36 \times 36$ grids. On this problem, we will show that our generic framework allows us to easily define local search algorithms and to combine and compare them.

\subsection{CSP Model}

Consider a $n^{2} \times n^{2}$ problem, an instinctive formalization considers a set of $n^{4}$ variables whose correspond, to all the cells to fill in. Using this, the set of related constraints is defined by AllDiff global contraints 8 representing : all digits appears only once in each row, once in each column and once in each $n \times n$ square the grid has been subdivided.

This model formalizes Sudoku problems in such a way that the CSP to solve has, for instance, 1296 variables and 108 constraints for the $36 \times 36$ grid. Usual approaches consider grids with a subset of cells already filled in and complete it (i.e., the real game for players), or aim at generating grids such that only one solution can be reach. In this paper we consider empty grids and our goal is to generate full ones. Even if Constraint Programming is useful for small problems, complete techniques are intractable for bigger instances. Indeed, grids with already filled positions (i.e., instantiated variables) are easier and we want to attack larger and more difficult problem since we consider local search strategies because of their ability in solving large scale problems.

We consider only grids such that all different digits appear once in each $n \times n$ sub-square. This corresponds to enforce some constraints of the problem directly in the encoding structure and it implies a restriction of the neighborhood to all possible swaps between cells of a same square. The eval function is related to the notion of solution and is defined for each constraint alldiff $c$ as a cost of violation (number of assignments to correct to satisfy the constraint). It is equal to 0 if the constraint is satisfied.

Concerning LS methods, on one hand, Tabu search (TS) 4 has been successfully applied to solve CSP [5]. Basically, this algorithm forbids moving to a sample that was visited less than $l$ steps before. To this end, the list of the last $l$ visited samples is memorized. On the other hand, we consider a basic descent technique with random walks RW where random moves are performed according to a certain probability $p$. According to our model, we only have now to design functions of the generic algorithm of Section 4.1 to model strategies. Neighborhood functions are functions $C \rightarrow C$ such that $(p, V) \mapsto\left(p, V \cup V^{\prime}\right)$ with different conditions: 
FullNeighbor: $V^{\prime}=\{s \in D \mid s \notin V\}$

TabuNeighbor: $V^{\prime}=\left\{s \in D \mid \nexists k, n-l \leq k \leq n, s_{k}=s\right\}$

DescentNeighbor : $p=\left(s_{1}, \ldots, s_{n}\right)$ and $V^{\prime}=s \subset D$ s.t. $\nexists s^{\prime} \in V$ s.t $\operatorname{eval}\left(s^{\prime}\right)<$ $\operatorname{eval}\left(s_{n}\right)$

Move functions are functions $C \rightarrow C$ such that $(p, V) \mapsto\left(p^{\prime}, \emptyset\right)$ with different conditions:

BestMove $: p^{\prime}=p \oplus s^{\prime}$ and $\operatorname{eval}\left(s^{\prime}\right)=\min _{s^{\prime \prime} \in V} \operatorname{eval}\left(s^{\prime \prime}\right)$.

Improve Move : $p=p^{\prime \prime} \oplus s_{n}$ and $p^{\prime}=p \oplus s$ s.t. $\operatorname{eval}\left(s^{\prime}\right)<\operatorname{eval}\left(s_{n}\right)$.

RandomMove : $p^{\prime}=p \oplus s^{\prime}$ and $s^{\prime} \in V$.

We can precise here the input set of function $F$ for algorithm GI.

Tabusearch : $\{$ TabuNeighbor; BestNeighBor $\}$

Randomwalk : \{FullNeighbor; BestNeighBor; RandomNeighbor $\}$

TabuSearch + Descent : $\{$ TabuNeighbor; DescentNeighbor

; ImproveNeighBor; BestNeighBor $\}$

Randomwalk + Descent : $\{$ FullNeighbor; BestNeighBor

; RandomNeighbor; DescentNeighbor

; ImproveNeighBor\}

Remark that the different algorithms correspond here to different sets of input functions and different behaviours of the choose function in the GI algorithm. This choose function select alternatively neighborhood function and move functions. For the Random Walk algorithm, given a probability parameter $p$, we have to introduce a quota of $p$ BestMove functions and $1-p$ RandomMove used in GI. Concerning Tabu Search, we use here a TabuNeighbor with $l=10$ and BestMove functions to built our Tabu Search algorithm. At last, we combine a descent strategy by adding DescentNeighbor and ImproveMove to the previous sets in order to design algorithms in which a Descent is first applied in order to reach more quickly to a good configuration.

\subsection{Experimentation Results}

In Table 1, we compare results of the tabu search and random walks associated with descent on different instances of Sudoku problem. Note that we have evaluated the difficulty of the problem thanks to a classic complete method with propagation and split, we obtained a more than one day cpu time cost for a $36 \times 36$ grid. At the opposite, by a simple formalization of the problem and thanks to a function application model, we are able to reach to a solution with classical local search algorithms and this starting from an empty grid. For each method and for each instance, 2000 runs have been performed except for $36 \times 36$ problem, 500 runs. Results we obtain by adding descent in a Tabu Search or in a Random Walk method, allows us to reduce the computation time to reach a solution. We may remark that the hybrid strategies combining several move and neighborhood functions provide better results. Our framework allows us to tune easily the balance between the different basic functions that characterize basic solving strategies and thus to design various algorithms in a single generic algorithm, which is not so clear if the methods are considered from a pure algorithmic point of view. 
Table 1. Results of Sudoku problem by different search approaches

\begin{tabular}{|c|c|c|c|c|c|c|}
\hline & \multicolumn{3}{|c|}{ TabuSearch } & \multicolumn{3}{c|}{ RandomWalk } \\
\hline$n^{2} \times n^{2}$ & $16 \times 16$ & $25 \times 25$ & $36 \times 36$ & $16 \times 16$ & $25 \times 25$ & $36 \times 36$ \\
\hline cpu time Avg & 3,14 & 115,08 & 3289,8 & 3,92 & 105,22 & 2495 \\
\hline deviations & 1,28 & 52,3 & 1347,4 & 1,47 & 49,3 & 1099 \\
\hline mvts Avg & 405 & 3240 & 22333 & 443 & 2318 & 13975 \\
\hline & Descent + TabuSearch & Descent + RandomWalk \\
\hline$n^{2} \times n^{2}$ & $16 \times 16$ & $25 \times 25$ & $36 \times 36$ & $16 \times 16$ & $25 \times 25$ & $36 \times 36$ \\
\hline cpu time Avg & 2,34 & 111,81 & 2948 & 2,41 & 82,94 & 2455 \\
\hline deviations & 1,42 & 55,04 & 1476 & 1,11 & 36,99 & 1092 \\
\hline mvts Avg & 534 & 3666 & 20878 & 544 & 2581 & 14908 \\
\hline
\end{tabular}

\section{Conclusion}

In this paper, we have proposed a framework for modeling CSP resolution with local search techniques. This framework provides a computational model as the computation of a fixed point of functions over a partial ordering, inspired the initial works of K.R. Apt [2]. The generic algorithm has been used to solve the Sudoku problem and allow us to compare different resolution approach in a very uniform framework where strategies can be easily designed.

This framework could be extended in order to include complete resolution mechanisms (constraint propagation, domain splitting) and even other metaheuristics such as evolutionary algorithms. It could also be used for experimental studies as it provides a uniform description framework for various methods in an hybridization context.

\section{References}

1. E. Aarts and J.K. Lenstra, editors. Local Search in Combinatorial Optimization. John Wiley and Sons, 1997.

2. K. Apt. From chaotic iteration to constraint propagation. In ICALP '97, number 1256 in LNCS, pages 36-55. Springer, 1997. invited lecture.

3. F. Focacci, F. Laburthe, and A. Lodi. Local search and constraint programming. In Handbook of Metaheuristics, Kluwer, 2002.

4. F. Glover and M. Laguna. Tabu Search. Kluwer Academic Publishers, 1997.

5. J.K. Hao and R. Dorne. Empirical studies of heuristics local search for constraint solving. In CP'96 LNCS 1118, pages 184-208, 1996.

6. N. Jussien and O. Lhomme. Local search with constraint propagation and conflictbased heuristics. Artificial Intelligence, 139(1):21-45, 2002.

7. E. Monfroy, F. Saubion, and T. Lambert. On hybridization of local search and constraint propagation. In ICLP 2004, number 3132 in LNCS, pages 180-194, 2004.

8. J.C. Régin. A filtering algorithm for constraint of difference in csps. In National Conference of Artificial Intelligence, pages 362-367, 1994. 